\title{
Micromagnetic Simulation of Domain Walls in Exchange Spring Trilayers
}

\author{
Zijun Wang, ${ }^{1}$ Xingqiao Ma, ${ }^{2}$ Houbing Huang, ${ }^{2}$ Hongwen Xiao, ${ }^{1}$ and Tianfu Li ${ }^{1}$ \\ ${ }^{1}$ China Institute of Atomic Energy, Xinzhen Street, Fangshan District, Beijing 102413, China \\ ${ }^{2}$ Department of Physics, University of Science and Technology Beijing, No. 30 Xueyuan Road, Haidian District, Beijing 100083, China \\ Correspondence should be addressed to Xingqiao Ma; xqma@sas.ustb.edu.cn
}

Received 14 December 2013; Revised 6 March 2014; Accepted 7 March 2014; Published 8 May 2014

Academic Editor: Hanxing Zhu

Copyright (c) 2014 Zijun Wang et al. This is an open access article distributed under the Creative Commons Attribution License, which permits unrestricted use, distribution, and reproduction in any medium, provided the original work is properly cited.

\begin{abstract}
Chiral domain wall structures in ferromagnetic exchange spring soft/hard/soft and hard/soft/hard trilayers were investigated with micromagnetic simulation, which enables us to fully characterize the nucleation and growth of buried domain walls in layered ferromagnetic thin films. Simulated results show that the trilayers are both exchange coupled and presenting chiral spin structures. Detailed features of field-dependent domain walls evolution in the spring magnets are also revealed. In process of remagnetization, the spin structure of soft/hard/soft is energetically more stable than that of hard/soft/hard.
\end{abstract}

\section{Introduction}

In the past decades, there has been a tremendous increase in the investigation of magnetic properties of exchange-coupled systems. Exchange-coupled multilayers with alternating hard and soft magnetic layers are of great technological and scientific interest. They can be used as permanent magnets with giant magnetic energy production $[1,2]$ or as giant magnetostrictive materials with low saturation field $[3,4]$. It is expected that the magnetic moments of soft magnetic layer rotate reversibly with the directions distributed successively depending on the distance from the hard/soft interface during the magnetization reversal process. This is the socalled exchange spring phenomenon [5].

A variety of studies have been carried out for layered exchange springs. The magnetization reversal process in exchange spring FeTaN/FeSm/FeTaN multilayers was investigated using magneto-optical Kerr effect vector magnetometry to measure in-plane hysteresis loops both parallel and perpendicular to the applied field [6-8]. Nucleation, compression, and propagation of $180^{\circ}$ domain walls in $\mathrm{GdFe} / \mathrm{TbFe} / \mathrm{GdFe}$ trilayers were presented. The behavior of domain walls was followed from the electric resistivity of the sample [9]. NiFe/SmFe/NiFe trilayers with in-plane uniaxial anisotropy induced by an applied magnetic field during deposition were prepared on (100)-Si substrates by dc magnetron sputtering. Magnetization hysteresis loops were measured by an alternating-gradient magnetometer for various angles between the external magnetic field and the easy axis [10]. The proposed $\mathrm{FePt} / \mathrm{Fe}_{3} \mathrm{Pt} / \mathrm{FePt}$ trilayer reduced the coercive field of a single-phase FePt of the same thickness [11]. FePt/Fe perpendicular exchange-coupled bilayers with different Fe thicknesses were prepared to study the exchange coupling effect and the magnetization switching mechanism [12]. Tuning exchange spring effects in $\mathrm{FePt} / \mathrm{Fe}(\mathrm{Co})$ magnetic bilayers, possibilities to maximize the exchange spring effects via suitably chosen nonhomogeneous soft magnetic layers are open [13].

Features of spin-dependent transport in many systems are related to the growth and movement of domain walls. The development of magnetic recording devices can be accelerated once we understand the physics of confined domain walls. Nevertheless, no magnetic imaging method can yield complete information about the three-dimensional magnetization distribution in a given sample. Techniques to image the moment, such as scanning electron microscopy with polarization analysis (SEMPA) [14], magneto-optical Kerr effect (MOKE) [15], and magneto-optical indicator film (MOIF) imaging [16], are surface sensitive and/or average the moment over the thickness of the magnetic modulations. 


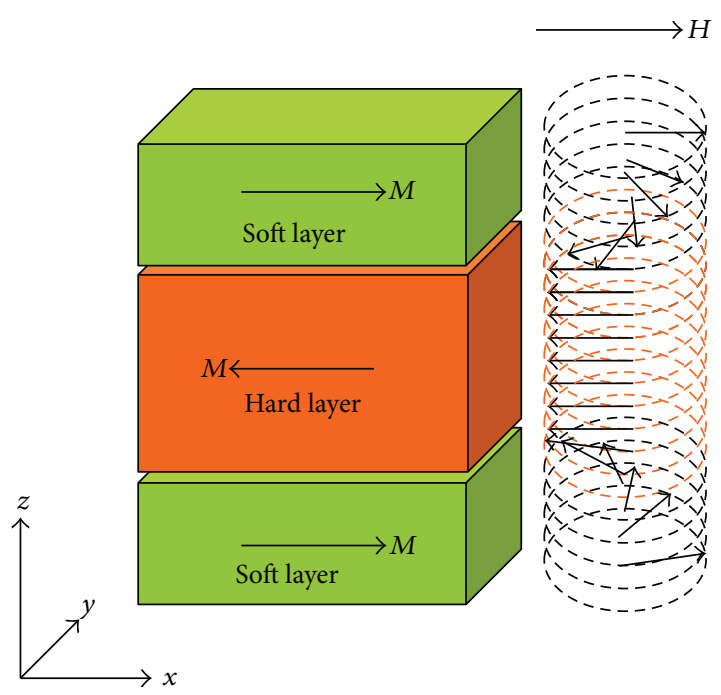

(a)
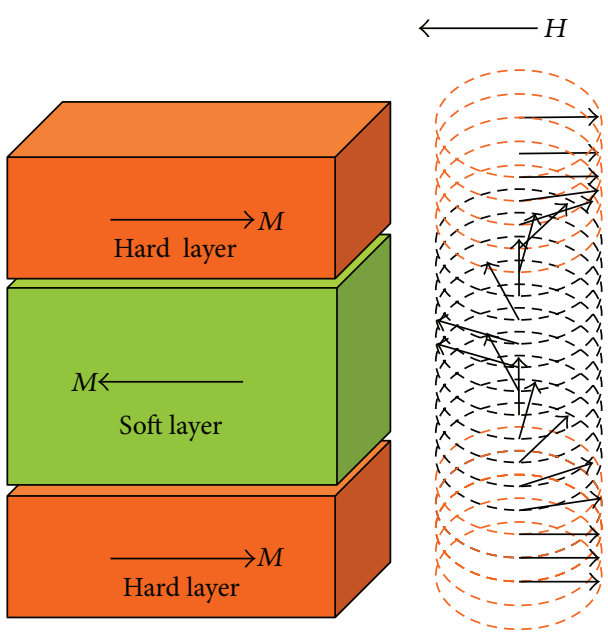

(b)

FIGURE 1: Geometry of (a) soft/hard/soft and (b) hard/soft/hard spring trilayer nanostructures and their magnetic domain wall structures.

This restriction can be overcome by computer simulation on microstructure evolving. Here we apply the microscopic phase field method, which is based on Ginzburg-Landau dynamics model, to simulate the magnetic domain walls evolving process with external field in two ferromagnetic exchange spring trilayer thin films [16]. In order to solve the nonlinear Landau-Lifshitz-Gilbert equations efficiently, Fortran computational advanced language was adopted.

\section{Theoretical Model}

The three-dimensional micromagnetic simulations are carried out by solving the Landau-Lifshitz-Gilbert (LLG) equations numerically:

$$
\begin{aligned}
\left(1+\alpha^{2}\right) \frac{\partial \vec{M}(r)}{\partial t}= & -\gamma_{0} \vec{M}(r) \times \vec{H}_{\mathrm{eff}} \\
& -\frac{\gamma_{0} \alpha}{M_{s}} \vec{M}(r) \times\left(\vec{M}(r) \times \vec{H}_{\mathrm{eff}}\right),
\end{aligned}
$$

where $\alpha$ is the damping constant, $\gamma_{0}$ is the gyromagnetic ratio, $M_{s}$ is the saturation magnetization, $\vec{M}$ is the magnetization, and $\vec{H}_{\text {eff }}$ is the effective magnetic field,

$$
\vec{H}_{\text {eff }}=-\frac{1}{\mu_{0}} \frac{\partial E}{\partial \vec{M}(r)},
$$

where $\mu_{0}$ is the permeability of vacuum and $E$ is the total free energy in magnets.

The total free energy of the bilayered exchange spring system is given by

$$
E=E_{\text {ani }}+E_{\text {ms }}+E_{\text {exch }}+E_{\text {external }}
$$

where $E_{\text {ani }}, E_{\mathrm{ms}}, E_{\text {exch }}$, and $E_{\text {external }}$ are magnetocrystalline anisotropy energy, magnetostatic energy, exchange energy, and Zeeman energy, respectively. The detailed expressions of the total free energy are

$$
\begin{aligned}
E= & \int\left(\frac{K_{j}}{M_{s j}{ }^{2}}\left\{(\vec{M} \cdot \widehat{u})^{2}\right\}\right) d^{3} r+\int \vec{H}_{\mathrm{dem}} \cdot \vec{M} d^{3} r \\
& +\frac{A}{M_{s j}{ }^{2}} \int[\operatorname{grad} \vec{M}(r)]^{2} d^{3} r-\int \vec{H}_{\mathrm{ext}} \cdot \vec{M} d^{3} r,
\end{aligned}
$$

where $\widehat{u}$ is a unit vector along the anisotropy axis, $K_{j}$ is the magnetic anisotropy constant, and $\vec{M}$ is the magnetization of the bilayer of magnitude $M_{s j} \cdot \vec{H}_{\mathrm{dem}}$ is the demagnetization field, $\vec{H}_{\text {ext }}$ is the external magnetic field, and the interface coupling $A_{I}$ is assumed to be equal to the exchange coefficient A.

Simulated structures include two different trilayer ferromagnets constitute of soft/hard/soft and hard/soft/hard thin films, as shown in Figure 1(a). The uppermost and lowest layers are soft ferromagnets. Both of their thicknesses are $44.5 \mathrm{~nm}$. The middle layer is hard layer with thickness of $89 \mathrm{~nm}$. Similar to soft/hard/soft trilayer model, the hard/soft/hard exchange spring is constituted by three thin films as showed in Figure 1(b), substituting the soft layers with hard ones and hard layer with soft one, respectively. The dynamics of magnetization was investigated by numerically solving the time-dependent LLG equation using the GaussSeidel projection method [17-19] with a constant time step $\Delta t=0.0238993$ ps [20]. Simulations with a shorter time step gave the same results exactly. The samples were discrete in computational cells of $2 \times 2 \times 2 \mathrm{~nm}^{3}$. Other parameters are adopted as shown in the following: soft layer saturation magnetization $M_{s_{1}}=10^{6} \mathrm{~A} / \mathrm{m}$, soft layer magnetic anisotropy constant $K_{s}=5 \times 10^{2} \mathrm{~J} / \mathrm{m}^{3}$, soft layer exchange constant $A_{s}=10^{-11} \mathrm{~J} / \mathrm{m}$; hard layer saturation magnetization $M_{s_{2}}=10^{6} \mathrm{~A} / \mathrm{m}$, hard layer magnetic anisotropy constant 


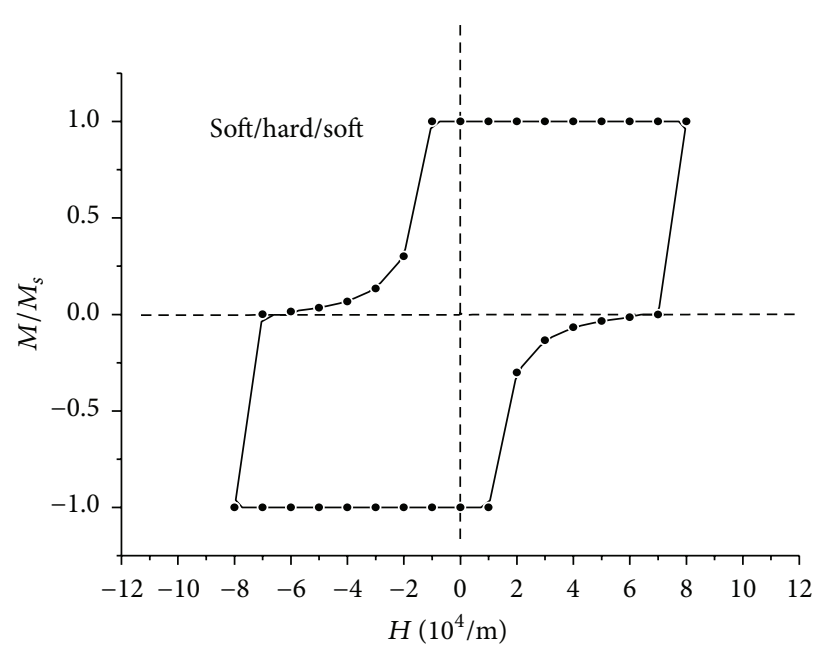

(a)

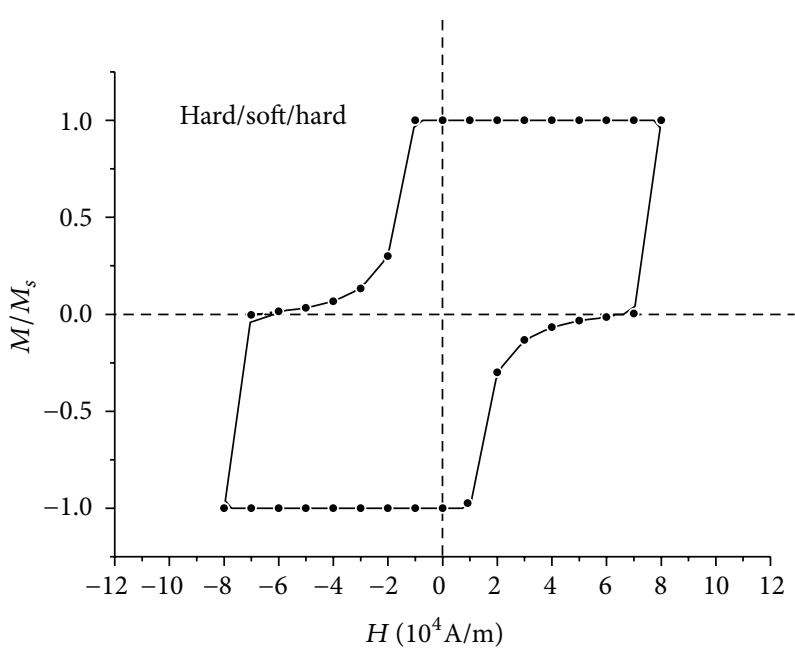

(b)

FIGURE 2: Hysteresis loops of (a) soft/hard/soft and (b) hard/soft/hard spring trilayers.

TABLE 1: Magnetization of hard/soft/hard and soft/hard/soft at different external magnetic fields.

\begin{tabular}{lcccccccc}
\hline$H(\mathrm{~A} / \mathrm{m})$ materials & $1.0 \times 10^{4}$ & $2.0 \times 10^{4}$ & $3.0 \times 10^{4}$ & $4.0 \times 10^{4}$ & $5.0 \times 10^{4}$ & $6.0 \times 10^{4}$ & $7.0 \times 10^{4}$ & $8.0 \times 10^{4}$ \\
\hline Hard/soft/hard & 1 & 0.3004 & 0.1333 & 0.0664 & 0.0335 & 0.0145 & -0.0030 & -1 \\
Soft/hard/soft & 1 & 0.3006 & 0.1336 & 0.0667 & 0.0339 & 0.0153 & 0.0003 & -1 \\
\hline
\end{tabular}

$K_{h}=10^{5} \mathrm{~J} / \mathrm{m}^{3}$, and hard layer exchange constant $A_{h}=$ $10^{-11} \mathrm{~J} / \mathrm{m}$. According to our previous calculations [21], the anisotropy constant of soft layer has a strong influence on the magnetic properties at a small applied field. However, for a large applied field, the magnetic properties are mainly influenced by anisotropy constant of hard layers.

\section{Results}

We investigated two different magnetic structures in exchange spring trilayers of soft $44.5 \mathrm{~nm} /$ hard $89 \mathrm{~nm} / \mathrm{soft}$ $44.5 \mathrm{~nm}$ and hard $44.5 \mathrm{~nm} / \mathrm{soft} 89 \mathrm{~nm} /$ hard $44.5 \mathrm{~nm}$ by using micromagnetic simulation. Schematic magnetization domain wall configurations of the two spring trilayers are showed on the right of Figures 1(a) and 1(b). The magnetic states of the samples were firstly saturation magnetized along $-x$-axis for (a) and $+x$-axis for (b), ensuring the uniform magnetization in all three layers of two structures. Then, the applied magnetic field was gradually increased along the $+x$ axis for (a) and $-x$-axis for (b). Micromagnetic simulation results indicate that the magnetic moments in the springs parallel the film plane and rotate successively with depth. As a result, the moments in each trilayer distribute as a chiral structure like Bloch domain walls. However, the two spin structures are intrinsic distinction excluding the effect of their different chemical configuration. The spin structure of soft/hard/soft distributes like a $360^{\circ}$ Bloch domain wall while that of hard/soft/hard like two $180^{\circ}$ Bloch domain walls.

Figures 2(a) and 2(b) show the in-plane hysteresis loops along $x$-axis for soft/hard/soft and hard/soft/hard spring trilayers, respectively. The "knee" shape of hysteresis loops is clearly observed, which indicates that both of the trilayers are exchange spring coupled. They show similar magnetic properties; for example, the two nucleation fields are nearly $1.0 \times 10^{4} \mathrm{~A} / \mathrm{m}$ and the irreversible switching fields are less than $8.0 \times 10^{4} \mathrm{~A} / \mathrm{m}$. From this point of view, they could be the potential materials for magnetostriction or permanent magnet with high performance. However, there is slight difference of hysteresis loops between them, and we will discuss it in Table 1.

The difference of the values of saturation magnetization between them is awfully small, which can be seen by the two nearly identical loops. To observe the difference between their saturation clearly, we present the concrete values as displayed in Table 1. From the table, the difference is of the magnitude of $10^{-4}$. As the external magnetic field increases, it becomes larger. For instance, it is 0.0002 at $2 \times 10^{4} \mathrm{~A} / \mathrm{m}$ and 0.0033 at $7 \times 10^{4} \mathrm{~A} / \mathrm{m}$. This is the case until the external magnetic field reaches $8 \times 10^{4} \mathrm{~A} / \mathrm{m}$, at which the two trilayers were both saturation magnetized. For each $H_{\text {ext }}$ between $2 \times 10^{4} \mathrm{~A} / \mathrm{m}$ and $6 \times 10^{4} \mathrm{~A} / \mathrm{m}$, the value of soft/hard/soft trilayer is always slightly larger than that of hard/soft/hard one, which means that the moments of the latter rotate more slowly than those of the former with $H_{\text {ext }}$ increased. In other words, the soft/hard/soft spring magnet is more energetically stable compared to the hard/soft/hard one. That soft/hard/soft magnetic moments are harder to change under $H_{\text {ext }}$ also indicates that the trilayer possesses stronger exchange-coupled effect.

To further elucidate the magnetic structure of the exchange spring trilayers, we divide the spring systems into 


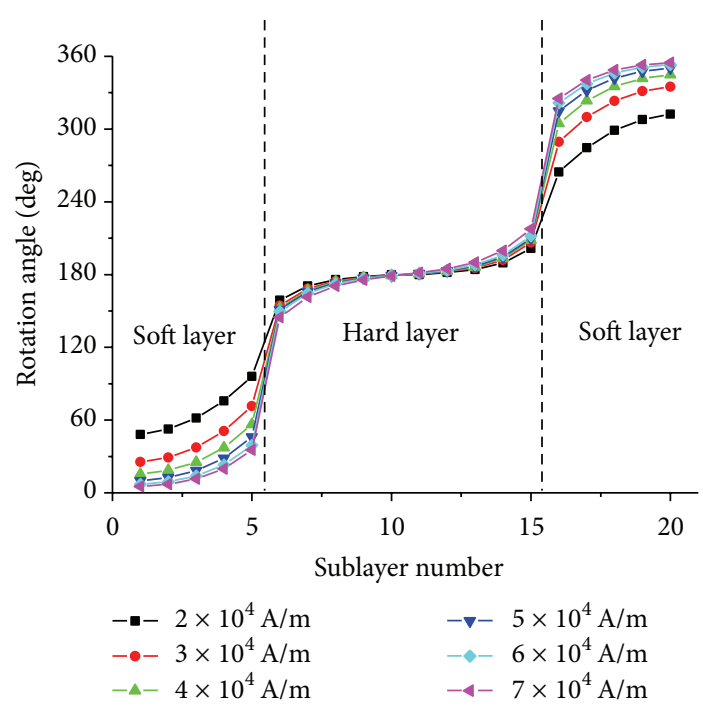

(a)

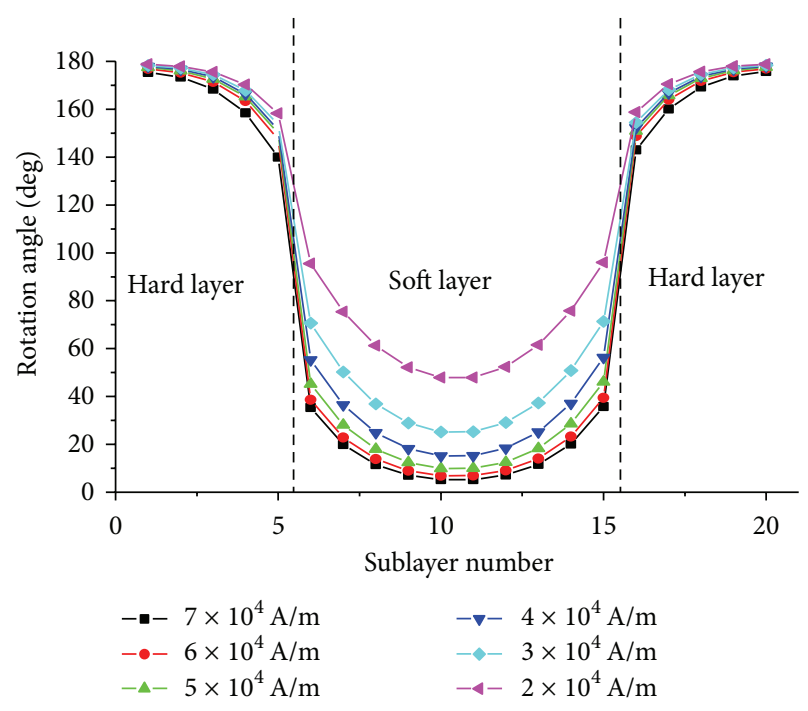

(b)

Figure 3: The angles of the magnetization to external field in (a) soft/hard/soft and (b) hard/soft/hard structures.

20 sublayers horizontally from up to down. Each sublayer is assumed to have a uniform direction of moment. The angles between magnetic moments and the field direction under different magnitude of magnetic fields are showed in Figure 3 $\left(2.0 \times 10^{4} \mathrm{~A} / \mathrm{m}, 3.0 \times 10^{4} \mathrm{~A} / \mathrm{m}, 4.0 \times 10^{4} \mathrm{~A} / \mathrm{m}, 5.0 \times 10^{4} \mathrm{~A} / \mathrm{m}\right.$, $\left.6.0 \times 10^{4} \mathrm{~A} / \mathrm{m}, 7.0 \times 10^{4} \mathrm{~A} / \mathrm{m}\right)$. Each curve corresponds to one point on the hysteresis loops.

The moments in soft layers play an important role during the process of remagnetization. These moments can be switched much more considerably than those moments in hard layers, from which we can safely infer that remagnetization nucleation firstly formed in soft layers and gradually propagated to hard ones. Contrary to our expectations for low field, reversible magnetization involves almost completely the hard ferromagnets. Therefore, the ranges of moments rotation angle are $0^{\circ} \sim 360^{\circ}$ and $0^{\circ} \sim 180^{\circ}$ for soft/hard/soft and hard/soft/hard, respectively. The moments in the two spring magnets are distributed as chiral structure which conforms to the principle of minimum energy.

The magnetization vectors distribution with depth expressed in degrees is showed in Figure 3. For soft/hard/soft, the rotation angles increase from surface to bottom, while for hard/soft/hard, the angles increase from the middle to either surface or bottom. For both trilayers, the rotation angles change successively with depth and the angular slopes increase with close to the interfaces (denoted by four vertical dashed lines). This tendency is drastically enlarged at the interfaces, suggesting exchange-coupled interaction there called energy barrier. With external field $H$ increased, the angle difference between two magnetization vectors at the interface (one vector in soft layer and the other one locating in hard layer) becomes larger significantly, leading to an increase of energy barrier. Of course, there is more or less interfacial roughness or diffusion for thin films in practical situation, brought by the film growth, which might weaken the barrier effect.

\section{Conclusions}

In summary, we investigated two different chiral structures of exchange-coupled soft/hard/soft and hard/soft/hard trilayers with micromagnetic simulation, which makes us readily extract the magnetic domain walls distribution with depth in buried layers. The two spring magnets have almost similar hysteresis loops. Nevertheless, they show intrinsic different domain wall structures. The hard/soft/hard one forms two $180^{\circ}$ Bloch domain walls while the soft/hard/soft one forms a $360^{\circ}$ Bloch wall due to the exchange-coupled effect. We present both the angle and angular gradient of the moments with film depth; thus the chirality of the whole spin structures is clearly obtained.

\section{Conflict of Interests}

The authors declare that there is no conflict of interests regarding the publication of this paper.

\section{Acknowledgment}

This work was sponsored by the National Science Foundation of China (11275274, 11005159, and 11174030).

\section{References}

[1] R. Skomski and J. M. D. Coey, "Giant energy product in nanostructured two-phase magnets," Physical Review B: Condensed Matter and Materials Physics, vol. 48, no. 21, pp. 15812-15816, 1993.

[2] E. E. Fullerton, J. S. Jiang, M. Grimsditch, C. H. Sowers, and S. D. Bader, "Exchange-spring behavior in epitaxial hard/soft magnetic bilayers," Physical Review B: Condensed Matter and Materials Physics, vol. 58, no. 18, pp. 12193-12200, 1998. 
[3] N. H. Duc, D. T. H. Giang, and N. Chau, "Novel exchangespring configuration for excellent magnetic and magnetostrictive softness," Journal of Magnetism and Magnetic Materials, vol. 290-291, part 2, pp. 800-803, 2005.

[4] H. D. Chopra, M. R. Sullivan, A. Ludwig, and E. Quandt, "Magnetoelastic and magnetostatic interactions in exchange-spring multilayers," Physical Review B: Condensed Matter and Materials Physics, vol. 72, no. 5, Article ID 054415, 7 pages, 2005.

[5] E. F. Kneller and R. Hawig, "The exchange-spring magnet: a new material principle for permanent magnets," IEEE Transactions on Magnetics, vol. 27, no. 4, pp. 3588-3595, 1991.

[6] G. Gubbiotti, P. Vavassori, G. Carlotti et al., "Structural and magnetic properties of exchange-spring FeTaN/FeSm/FeTaN multilayers," Surface Science, vol. 566-568, part 1, pp. 285-290, 2004.

[7] G. Gubbiotti, G. Carlotti, J. Weston, G. Zangari, D. C. Crew, and R. L. Stamps, "Asymmetry in the static and dynamic magnetic properties of a weak exchange spring trilayer," Journal of Magnetism and Magnetic Materials, vol. 286, pp. 479-483, 2005.

[8] P. Vavassori, G. Gubbiotti, G. Carlotti et al., "Magnetization reversal in exchange-coupled FeTaN/FeSm/FeTaN multilayers," Journal of Magnetism and Magnetic Materials, vol. 272-276, supplement 1, pp. E949-E950, 2004.

[9] S. Mangin, G. Marchal, C. Bellouard, W. Wernsdorfer, and B. Barbara, "Magnetic behavior and resistivity of the domain-wall

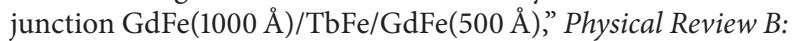
Condensed Matter and Materials Physics, vol. 58, no. 5, pp. 27482757, 1998.

[10] S.-S. Yan, W. J. Liu, J. L. Weston, G. Zangari, and J. A. Barnard, "Magnetization-reversal mechanism of hard/soft exchangecoupled trilayers," Physical Review B: Condensed Matter and Materials Physics, vol. 63, no. 17, Article ID 174415, 7 pages, 2001.

[11] D. Suess, T. Schrefl, R. Dittrich et al., "Exchange spring recording media for areal densities up to $10 \mathrm{Tbit} / \mathrm{in}^{2}$," Journal of Magnetism and Magnetic Materials, vol. 290-291, part 1, pp. 551-554, 2005.

[12] L. S. Huang, J. F. Hu, and J. S. Chen, "Critical Fe thickness for effective coercivity reduction in $\mathrm{FePt} / \mathrm{Fe}$ exchange-coupled bilayer," Journal of Magnetism and Magnetic Materials, vol. 324, no. 6, pp. 1242-1247, 2012.

[13] V. R. Reddy, O. Crisan, A. Gupta, A. Banerjee, and V. Kuncser, "Tuning exchange spring effects in $\mathrm{FePt} / \mathrm{Fe}(\mathrm{Co})$ magnetic bilayers," Thin Solid Films, vol. 520, no. 6, pp. 2184-2189, 2012.

[14] J. Unguris, R. J. Celotta, and D. T. Pierce, "Observation of two different oscillation periods in the exchange coupling of $\mathrm{Fe} / \mathrm{Cr} / \mathrm{Fe}(100)$," Physical Review Letters, vol. 67, no. 1, pp. 140$143,1991$.

[15] O. Hellwig, J. B. Kortright, K. Takano, and E. E. Fullerton, "Switching behavior of $\mathrm{Fe}-\mathrm{Pt} / \mathrm{Ni}-\mathrm{Fe}$ exchange-spring films studied by resonant soft-x-ray magneto-optical Kerr effect," Physical Review B: Condensed Matter and Materials Physics, vol. 62, no. 17, pp. 11694-11698, 2000.

[16] J. S. Jiang, E. E. Fullerton, C. H. Sowers et al., "Spring magnet films," IEEE Transactions on Magnetics, vol. 35, no. 5, pp. 32293234, 1999.

[17] H. B. Huang, X. Q. Ma, Z. H. Liu, C. P. Zhao, S. Q. Shi, and L. Q. Chen, "Simulation of multilevel cell spin transfer switching in a full-Heusler alloy spin-valve nanopillar," Applied Physics Letters, vol. 102, no. 4, Article ID 042405, 2013.

[18] H. B. Huang, X. Q. Ma, Z. H. Liu et al., "Micromagnetic simulation of spin-transfer switching in a full-Heusler $\mathrm{Co}_{2} \mathrm{FeAl}_{0.5} \mathrm{Si}_{0.5}$ alloy spin-valve nanopillar," Journal of Applied Physics, vol. 110, no. 3, Article ID 033913, 2011.

[19] H. B. Huang, X. Q. Ma, Z. H. Liu, F. Y. Meng, S. Q. Shi, and L. Q. Chen, "Micromagnetic simulation of critical current density of spin transfer torque switching in a full-Heusler $\mathrm{Co}_{2} \mathrm{FeAl}_{0.5} \mathrm{Si}_{0.5}$ alloy spin valve nanopillar," Journal of Magnetism and Magnetic Materials, vol. 330, pp. 16-20, 2013.

[20] H. B. Huang, X. Q. Ma, Z. H. Liu, and L. Q. Chen, "Modelling high-power spin-torque oscillator with perpendicular magnetization in half-metallic Heusler alloy spin valve nanopillar," Journal of Alloys and Compounds, vol. 597, pp. 230-235, 2014.

[21] Z. J. Wang, P. P. Wu, M. M. Wu, and X. Q. Ma, "Micromagnetic simulation of magnetic hysteresis in hard/soft bilayered exchange spring magnets," Advanced Materials Research, vol. 785-786, pp. 684-689, 2013. 

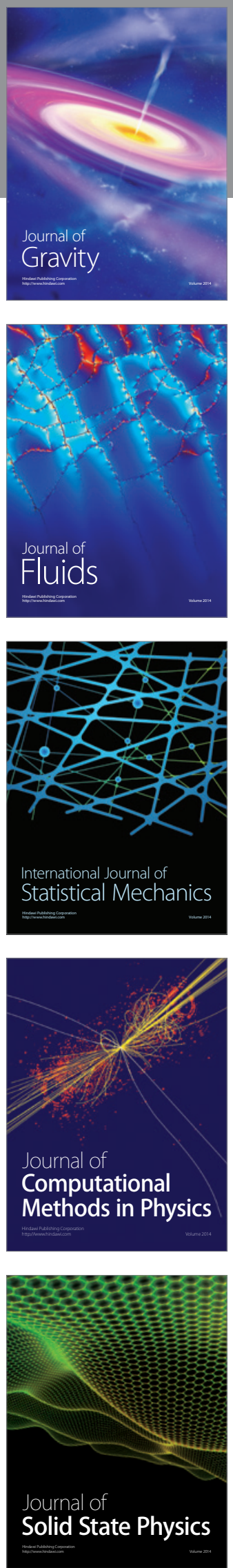

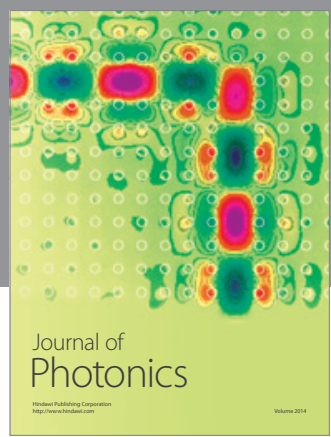

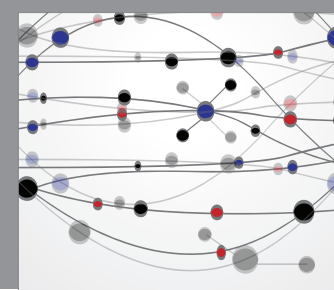

The Scientific World Journal

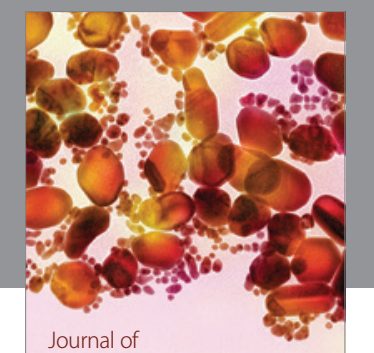

Soft Matter
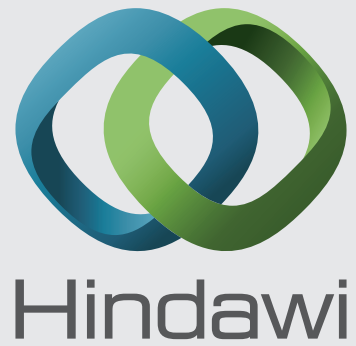

Submit your manuscripts at

http://www.hindawi.com
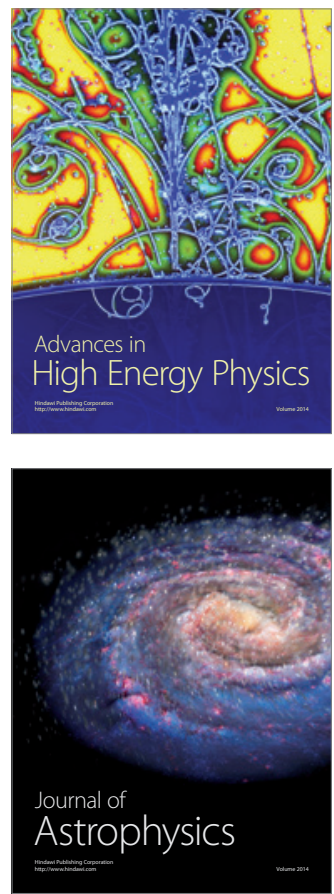
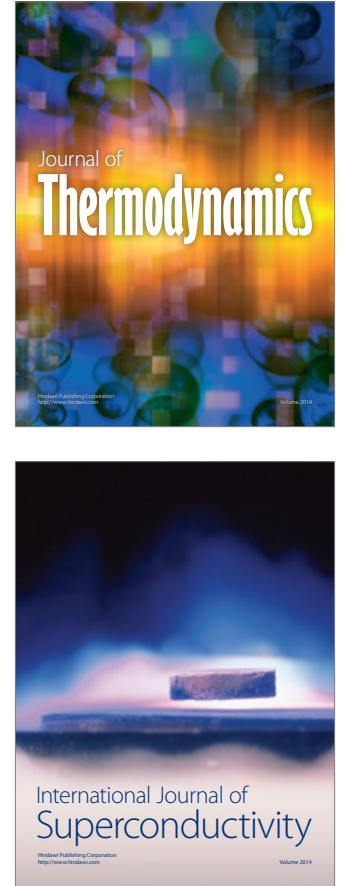
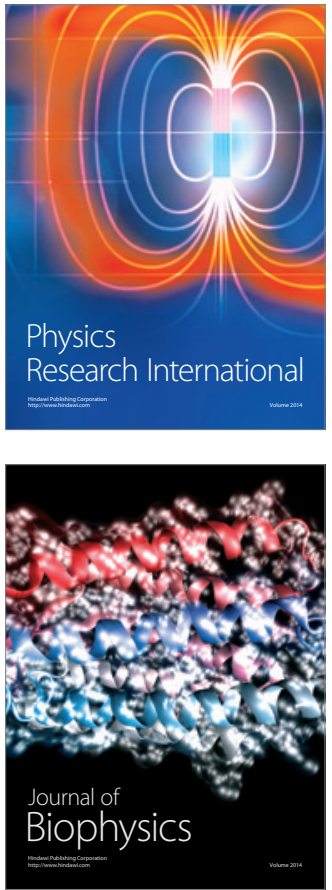
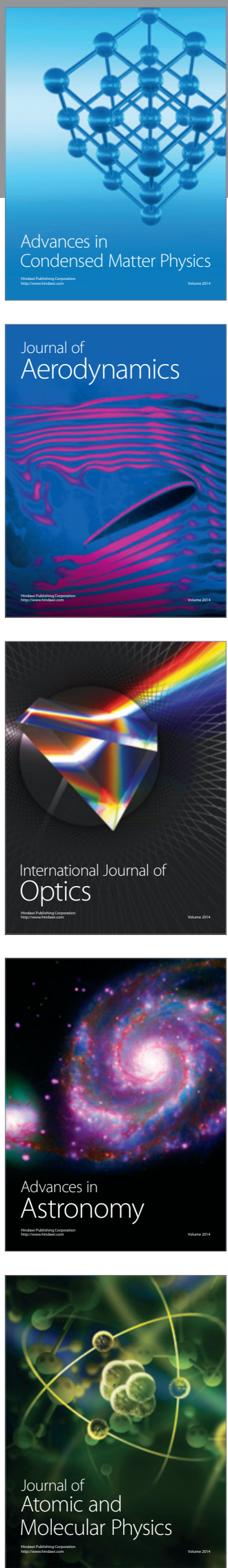